\title{
Causality in quantum mechanics
}

\author{
David T. Pegg
}

Centre for Quantum Computer Technology, School of Science, Griffith University, Nathan, Brisbane, Q 4111, Australia

\begin{abstract}
We show explicitly how the causal arrow of time that follows from quantum mechanics has already been inserted at a deeper level by the choice of normalisation conditions. This prohibits information being sent backwards in time but does not determine a time direction for state propagation.
\end{abstract}

PACS codes: 03.65.-w 03.65.Ta 03.65.Ud

Keywords: Causality, retrodiction, quantum measurement, retrocausation.

Corresponding author: Prof. D. T. Pegg, Centre for Quantum Computer Technology, School of Science, Griffith University, Nathan, Brisbane, Q 4111, Australia Phone: +61 $737357152 \quad$ Fax: +61 737357656

E-mail address: D.Pegg@griffith.edu.au 
The problem of the physical origin of the arrow of time is one of long standing [14]. Basic physics is essentially time symmetric but we observe a strong asymmetry in nature. We remember the past but not the future and we feel we have some control over future events but not over past events. The latter phenomenon is usually referred to as the principle of causality. Some authors [5] enunciate two causality principles: the strong and the weak. The strong principle states that a cause must always precede an effect, even at the microscopic level. In quantum mechanics this is manifest in assigning a state to a system based on an earlier preparation event and allowing the state to evolve forwards in time until it is collapsed by a subsequent measurement. The weak principle states that a controllable message cannot be sent backwards in time in any reference frame, that is, macroscopic information cannot be propagated faster than light. The weak principle is less stringent and does not require the strong principle to hold. Many arguments used to explain the origin of the arrow of time, such as trying to derive it from statistics alone, are fallacious with the time asymmetry that is meant to follow from the argument being inserted at an earlier stage [6]. Unfortunately the insertion point is not always obvious. Sometimes the insertion is by means of the language we use to ask the questions, giving them a hidden temporal bias $[3,6]$.

In this paper we examine causality in quantum mechanics. The well-known conventional view is that a closed quantum mechanical system evolves in two ways: smoothly with unitary evolution between preparation and measurement and discontinuously at the time of the measurement event. While the former is reversible, the latter is not so there is a common viewpoint that time asymmetry may have its roots in the quantum measurement process. This is perhaps most apparent in the many-worlds 
interpretation of quantum mechanics [7] in which the universe branches at each measurement event, giving an increasing number of worlds as time goes on. As quantum mechanics is so fundamental, it is natural to try to explain the physical origin of causality and the arrow of time in terms of it. It is not the intention of this paper to do so, however. Here we show explicitly how and where causality is subtly inserted into quantum mechanics rather than arising from it. This means that the origin of the arrow of time must be sought elsewhere. We also show that the formalism of quantum mechanics incorporates only the weak principle of causality, giving us some flexibility in interpreting standard quantum paradoxes.

A fundamental quantum postulate is that a description of nature can be obtained by use of mathematical operators acting on a Hilbert space. The states themselves can more generally be represented as density operators. To connect these mathematical operators to real measurement events in the laboratory, some probability postulate must also be invoked. The essential features of a quantum mechanical experiment are preparation of a system at some time, evolution and then measurement at a later time. We usually consider the prepared state as evolving with time until it is measured, whereupon it collapses in accord with the measurement outcome. This forward time evolution assumption fits in with our notion of causality and it is tempting to consider this assumption to be the point at which causal asymmetry is inserted. This is not true, however, as we shall see below. To find out where the asymmetry associated with causality is inserted into our basic quantum postulates, we first consider the case in which the evolution is removed by allowing the interval between preparation and measurement to be much shorter than the characteristic evolution time. We must then be careful to 
avoid first inserting the asymmetry into our probability postulate. We proceed, therefore, by adopting a fundamental postulate that is symmetric in preparation and measurement.

We consider the situation where one person, the preparer, prepares a system in some state and sends a label $i$ representing the preparation event to a computer. She then repeats this procedure on a large number of identical systems, preparing any states she chooses. Later, but in a time much shorter than the evolution time, the measurer performs a measurement on each of the systems in the order in which they were prepared and sends each measurement outcome $j$ to the computer. The computer records the preparation event and the associated measurement event for each system and prepares a list of combined events $(i, j)$. The fundamental symmetric postulate for the probabilistic interpretation of quantum mechanics that we shall adopt is as follows: the probability, as measured by the occurrence frequency, that any combined event chosen at random from the list is a particular combined event $(i, j)$ is given by [8]

$$
P(i, j)=\frac{\operatorname{Tr}\left(\hat{\Lambda}_{i} \hat{\Gamma}_{j}\right)}{\operatorname{Tr}(\hat{\Lambda} \hat{\Gamma})}
$$

where $\hat{\Lambda}=\sum_{i} \hat{\Lambda}_{i}$ and $\hat{\Gamma}=\sum_{j} \hat{\Gamma}_{j}$. The operators $\hat{\Lambda}_{i}$ and $\hat{\Gamma}_{j}$, which act on the Hilbert space of the system, are positive or negative definite and are associated with preparation event $i$ and measurement event $j$. Our reasons for adopting this postulate, in addition to its symmetry, simplicity and the fact that it gives eventual results in accord with experience, are as follows. We need two operators to represent preparation and measurement. We take the product of these operators rather than, for example, the sum, as a parallel to the way in which we combine classical probabilities to obtain a probability of a combined event. The trace over the Hilbert space ensures that we extract 
a number that is independent of the order of two non-commuting operators, thereby maintaining the symmetry. The normalising denominator ensures that the probability is less than or equal to unity. The operators $\hat{\Lambda}_{i}$ and $\hat{\Gamma}_{j}$ must be positive or negative definite to ensure the probability is positive. These operators, which we refer to as preparation or measurement device operators, include a description of the state prepared or measured. The two complete sets of these operators can be regarded as mathematical representations of the actions of the preparation and measurement devices. Examples of positive definite operators include projection operators, density operators and elements of a probability operator measure. For the moment, however, we keep $\hat{\Lambda}_{i}$ and $\hat{\Gamma}_{j}$ as general as possible to maintain the symmetry. Later we shall see that the introduction of a causality postulate gives these operators more specific meanings.

From (1) we can easily calculate other probabilities either from simple probability theory or directly from occurrence frequencies. We find the probability for measurement event $i$ to be

$$
P(i)=\sum_{j} P(i, j)=\frac{\operatorname{Tr}\left(\hat{\Lambda}_{i} \hat{\Gamma}\right)}{\operatorname{Tr}(\hat{\Lambda} \hat{\Gamma})}
$$

Suppose the series of preparations takes, say, one hour and the measurer waits one day to do the measurements and the computer produces the list after this. $P(i)$ in (2) is a function of $\hat{\Gamma}$ which relates to the measuring device and the measurer can choose the type of measuring device used. If this ability to choose gives the measurer some control over $\hat{\Gamma}$, he would have some control over $P(i)$. Now $P(i)$ is equal to the probability that the preparer prepares a particular state, which the preparer can ascertain before looking at the list. Thus if the measurer could influence $P(i)$ by his choice of measuring device he 
could use this control to send a message to the preparer backwards in time in violation of weak causality. For example, if the system is a spin-half atom the measurer could alter the direction of the field in his Stern-Gerlach apparatus to change $\hat{\Gamma}_{j}$ to $\hat{R} \hat{\Gamma}_{j} \hat{R}^{\dagger}$ where $\hat{R}$ is a unitary rotation operator. Alternatively he could delay the time of the measurement. As can be seen from our later discussion including time evolution, the effect of this delay can be expressed as a unitary time translation of $\hat{\Gamma}_{j}$. In order to be unaffected by any such changes the measurer might make, and thus preserve weak causality, $\hat{\Gamma}$ must commute with all unitary operators that can produce a change in the individual measuring device operators. Also the measurer could decide to change the measuring apparatus or even not to make a measurement at all. If $\hat{\Gamma}$ is to be invariant under all changes described above it must be proportional to the unit operator, the only operator with these properties [9]. With $\hat{\Gamma}=k \hat{1}$ we find that (2) becomes simply

$$
P(i)=\frac{\operatorname{Tr} \hat{\Lambda}_{i}}{\operatorname{Tr} \hat{\Lambda}}
$$

which is clearly independent of the measuring device as required by weak causality. We could also prevent the preparer sending information to the measurer via the quantum channel by ensuring that $\hat{\Lambda} \propto \hat{1}$, but in accord with weak causality we do not wish to do this. Thus weak causality induces an asymmetry between $\hat{\Gamma}$ and $\hat{\Lambda}$.

From (1) we obtain the probability for measurement event $j$ if preparation event $i$ occurs as

$$
P(j \mid i)=\frac{P(i, j)}{P(i)}=\frac{\operatorname{Tr}\left(\hat{\Lambda}_{i} \hat{\Gamma}_{j}\right)}{\operatorname{Tr}\left(\hat{\Lambda}_{i} \hat{\Gamma}\right)} .
$$


It is useful to define a normalised set of measuring device operators $\hat{\Pi}_{j}=\hat{\Gamma}_{j} / k$. Then the weak causality condition $\hat{\Gamma}=k \hat{1}$ becomes

$$
\sum_{j} \hat{\Pi}_{j}=\hat{1}
$$

Using this condition in (4) gives

$$
P(j \mid i)=\frac{\operatorname{Tr}\left(\hat{\Lambda}_{i} \hat{\Pi}_{j}\right)}{\operatorname{Tr} \hat{\Lambda}_{i}}=\operatorname{Tr}\left(\hat{\rho}_{i} \hat{\Pi}_{j}\right)
$$

where $\hat{\rho}_{i}=\hat{\Lambda}_{i} / \operatorname{Tr} \hat{\Lambda}_{i}$ must be a positive definite operator with a trace of unity. It is thus a density operator. $\hat{\Pi}_{j}=\hat{\Gamma}_{j} / k$ must also be positive definite because, from the relation $\hat{\Gamma}=k \hat{1}, k$ is negative if $\hat{\Gamma}_{j}$ is negative definite. As the operators $\hat{\Pi}_{j}$ sum to the unit operator, they are elements of a probability operator measure (POM), or positive operator valued measure [10]. This introduced asymmetry in the normalisations of $\hat{\rho}_{i}$ and $\hat{\Pi}_{j}$ ensures weak causality. Expression (6) is just the usual (asymmetric) postulate connecting probability and quantum mechanics [10]. Thus we see that the usual postulate already includes weak causality and we have seen how it has been inserted.

Because $P(i)$ is independent of any subsequent measurement and even independent of whether there is a later measurement or not, we call $P(i)$ the a priori probability of the preparation event $i$. On the other hand, from (1) with the weak causality condition, we obtain

$$
P(j)=\frac{\operatorname{Tr}\left(\hat{\Lambda} \hat{\Pi}_{j}\right)}{\operatorname{Tr} \hat{\Lambda}}=\sum_{i} P(i) \operatorname{Tr}\left(\hat{\rho}_{i} \hat{\Pi}_{j}\right) .
$$


The contrast with (3) is evident. $P(j)$ is dependent on the $a$ priori probabilities for preparation events as well as on the construction of the measuring device. This expression is consistent with the measurer being able to choose his measuring device but, once this has been done, having no control over the individual measurement outcome.

For completeness we give the fourth probability expression derivable from (1). With the weak causality condition included, we find

$$
P(i \mid j)=\frac{P(i) \operatorname{Tr}\left(\hat{\rho}_{i} \hat{\Pi}_{j}\right)}{\sum_{i} P(i) \operatorname{Tr}\left(\hat{\rho}_{i} \hat{\Pi}_{j}\right)} .
$$

For the special case where $\hat{\Pi}_{j}$ is a pure state projection operator, (8) reduces to the form of the posterior probability that a state $\hat{\rho}_{i}$ was prepared given a particular measurement outcome $j$, as deduced by Peres [11] using Bayesian statistics and described by him as the only retrodictive form that can be legitimately derived from conventional quantum mechanics.

We now examine strong causality. Classically, if we prepare a gyroscope to point in the $z$-direction and then measure it after a short enough time, we must also find it to be pointing in the $z$-direction. What state do we infer that it was in between preparation and measurement? Here the answer is unambiguous: we can assign either the state we know was prepared or the state we know was measured. If we replace the gyroscope with a spin-half atom, however, we could prepare it with its spin in the $z$-direction and then measure it to have its spin in the $x$-direction. Here there is choice of assigning the prepared state density operator $|+z\rangle\langle+z|$ or the measured state density operator $|+x\rangle\langle+x|$ between preparation and measurement. Traditionally we assign the prepared 
state, a choice based on our notion of strong causality. Our picture is that of the prepared state evolving from the preparation time $t_{p}$ into the future until it collapses to the measured state at the measurement time $t_{m}$. Let us now assume that the difference between preparation and measurement is long enough for some unitary evolution of the system to take place. Traditionally we would then assign the prepared state $\hat{\rho}_{i}$ at time $t_{p}$ immediately following preparation and assume this evolves to $\hat{U}\left(t, t_{p}\right) \hat{\rho}_{i} \hat{U}^{\dagger}\left(t, t_{p}\right)$ at some later time $t$ before measurement, where the unitary time shift operator is determined by Schrödinger's time-dependent equation. Incorporating this time dependence into (6) gives

$$
P(j \mid i)=\operatorname{Tr}\left[\hat{U}\left(t_{m}, t_{p}\right) \hat{\rho}_{i} \hat{U}^{\dagger}\left(t_{m}, t_{p}\right) \hat{\Pi}_{j}\right] .
$$

This result has been verified by many experiments, which would seem to justify our assignment of the forward evolving prepared state. However, although this forward-time evolution tradition, which has its roots in our concept of strong causality, predicts correct experimental results, this is not sufficient justification for it. In the end we measure probabilities. Mathematically we can rewrite (9) equivalently as

$$
P(j \mid i)=\operatorname{Tr}\left[\hat{\rho}_{i} \hat{U}^{\dagger}\left(t_{m}, t_{p}\right) \hat{\Pi}_{j} \hat{U}\left(t_{m}, t_{p}\right)\right] .
$$

This can be deduced from the cyclic property of the trace or from the invariance of the trace under a unitary transformation. Expression (10) can be interpreted in terms of the measured state propagating backwards in time until it is projected onto the prepared state at the preparation time. (We note here we are referring to $\hat{\Pi}_{j}$ as a state. If we wish to reserve this term for a density operator, we can easily convert $\hat{\Pi}_{j}$ to a state by dividing 
by its trace. Of course when $\hat{\Pi}_{j}$ is a pure state projector, as is often the case, its trace is unity anyway so it is already a state.) This “retrocausal” interpretation yields the same probabilities and thus does not violate weak causality. Thus our attempt to insert strong causality by the traditional state assignment does not lead to any mathematical or physically measurable difference between the traditional causal and the retrocausal assignment. This leads us to conclude that causality in quantum mechanics is not manifest in the time direction of state propagation, it is ensured by the different normalisation conditions for the preparation and measurement device operators. The time direction of information propagation is unaffected by the time direction of state propagation provided the normalisation conditions are maintained. Weak causality is essential but extending this to strong causality as described here has no physical consequences.

The above result gives us the freedom to picture the state as propagating forwards in time from preparation or backwards in time from measurement. If used carefully, it even allows us to use both. This extra freedom to treat states as travelling backwards in time is particularly useful, even for open systems, for calculating retrodictive probabilities in order, for example, to retrodict the signal sent in a noisy quantum communication channel [12]. Interestingly, it also allows us to take a different view of some quantum paradoxes. We consider two such paradoxes here, with the first being the well-known Schrödinger's cat. Here a preparation device is set up so that there is an amplitude for the cat to be killed by gas and an amplitude for it to survive. The cat exists in a superposition state of being dead and alive until observation, or measurement, determines if it is dead or alive. In this traditional interpretation it is the act of 
measurement that collapses the cat to the dead state if it is found dead. In the alternative, and equally, valid picture, we assign to the cat a state $\hat{\Pi}_{j}$, which is the dead state if that is the measurement outcome. We then follow the evolution of the dead cat backwards in time until the point where it is gassed. Before that point it was alive. In this picture the cat is dead between gassing and measurement and it is the gassing associated with the preparation device that kills the cat and not the act of observation. Weak causality is fully preserved and we have a picture more in keeping with a classical picture involving macroscopic objects if that is what we prefer.

A second example is the Einstein-Rosen-Podolsky paradox as verified by experiments in which an atom decays via an intermediate state to the ground state, emitting two photons with correlated polarizations $[13,14]$. In the traditional interpretation, in which an entangled state propagates forwards in time from the atom, it appears that the choice of the polarizer orientation at the time of detection of one photon immediately affects the polarization of the other photon some sizeable distance away. This instantaneous transmission of the influence not only seems to violate causality, but it is also not Lorentz covariant. In the alternate picture [15] we assign a state to the first photon emitted on the basis of the appropriate POM element, which is dependent on the detector polarization. This state, with its specific polarization, travels back along the light cone to the atom and collapses the intermediate state of the atom to one that corresponds to that polarization. The second photon is subsequently emitted with a polarization corresponding to this intermediate state. We then follow the propagation of this state forwards on the light cone from the atom to the second detector. The probability of detection by the second detector is the same as that which results from the 
traditional entangled state calculation. The difference between this picture and the traditional picture is that the former is Lorentz covariant and the collapses are all local, allowing us to retain reality, locality and weak causality if that is what we prefer. This picture is reminiscent of the description in terms of advanced and retarded electromagnetic waves of absorber theory [5, 16].

In conclusion, it has been the purpose of this paper to show the explicit point at which causality, and thus the arrow of time, is inserted into conventional quantum mechanics. We have shown that this lies in the choice of different normalisation conditions for the positive definite operators representing the preparation and measurement device outcomes. Causality results in the preparation device outcome being represented by a positive operator with unit trace and the measurement device outcome being represented by a positive operator that is an element of a set whose elements sum to the unit operator. These normalisation conditions ensure the time direction of information propagation, that is, they ensure that information cannot be sent into the past. These conditions do not, however, determine the time direction of state propagation. We have the freedom to assign a state of a system in accord with either the preparation outcome or the measurement outcome. In the latter case the state propagates backwards in time from the measurement event to the preparation event. Such states still give the correct probabilities and, as long as the normalisation conditions are obeyed, cannot be used to send a message backwards in time. These states do, however, allow alternative descriptions of some quantum paradoxes which may be more suited to different individual preferences. 


\section{Acknowledgements}

I thank Stephen Barnett, John Jeffers and Kenny Pregnell for their help in discussions over the years. This work was supported financially by the Australian Research Council and the Queensland Government.

\section{References}

[1] T. Gold (Ed.), The Nature of Time, Cornell University Press, Ithaca, 1967.

[2] L. S. Schulman, Time’s Arrows and Quantum measurement, Cambridge University Press, Cambridge, 1997.

[3] S. F. Savitt (Ed), Time’s Arrows Today, Cambridge University Press, Cambridge, 1997. See particularly the article by H. Price.

[4] I. D. Novikov, The River of Time, University Press, Cambridge, 1998.

[5] J. C. Cramer, Phys. Rev. D 22 (1980) 362.

[6] D. T. Pegg, Contemporary Physics 39 (1998) 195.

[7] H. Everett, Rev. Mod. Phys. 29 (1957) 454.

[8] D. T. Pegg, S. M. Barnett and J. Jeffers, J. Mod. Opt. 49 (2002) 913.

[9] K. L. Pregnell, Retrodictive Quantum State Engineering, Ph.D. thesis, Griffith University, Brisbane, 2004.

[10] C. W. Helstrom, Quantum Detection and Estimation Theory, Academic Press, New York, 1976.

[11] A. Peres, Phys. Lett. A 203 (1995) 150. 
[12] S. M. Barnett, D. T. Pegg, J. Jeffers and O. Jedrkiewicz, Phys. Rev. Lett. 86 (2001) 2455.

[13] C. A. Kocher and E. D. Commins, Phys. Rev. Lett. 18 (1967) 575.

[14] A. Aspect, Phys. Rev. D 14 (1976) 1944.

[15] D. T. Pegg and S. M. Barnett, J. Opt. B: Quantum Semiclass. Opt. 1 (1999) 442.

[16] D. T. Pegg, Phys. Lett. 78A (1980) 233. 